\section{A unique case of metastatic spinal epidural abscess associated with liver abscess following ascending cholangitis and Escherichia coli bacteremia}

\author{
Nour Karra ${ }^{1 \#}$, Samer Ganam" ${ }^{1 \#}$ Amitai Bickel ${ }^{1,2}$, Maxim Bez ${ }^{3}$, \\ Ibrahim Abu Shakra', Doron Fischer ${ }^{4}$ and Eli Kakiashvili ${ }^{1 *}$ \\ ${ }^{1}$ Department of Surgery A, Galilee Medical Center, Nahariya, Israel \\ ${ }^{2}$ Faculty of Medicine, Galilee Bar Ilan University, Safed, Israel \\ ${ }^{3}$ Medical Corps, Israel Defence Forces, Ramat Gan, Israel \\ ${ }^{4}$ Department of Radiology, Galilee Medical Center, Nahariya, Israel \\ \#These authors are equally contributed
}

\section{Abstract}

Pyogenic liver abscess (PLA) is a life-threatening infection that may develop as a result of an underlying hepatobiliary disease. A possible complication of PLA is metastatic spread, resulting in distant seeding of infection in other organs, and occasionally in the epidural space. Spinal epidural abscess (SEA) is a rare infection with severe potential complications. We describe a 71-year-old patient who presented with ascending cholangitis that was complicated by micro PLA, with a subsequent Escherichia coli bacteremia and metastatic SEA. An emergent surgical intervention with laminotomy and drainage of the epidural collection was performed. The patient was treated with a prolonged antibiotic regimen, with uneventful recovery and no neurologic sequelae. To our knowledge, this is the first reported case of a SEA following E. coli PLA.

\section{More Information}

*Address for Correspondence: Eli Kakiashvili, Department of Surgery A, Galilee Medical Center, Nahariya, Israel, Tel: +972507887808; Fax: (9724)910-7736; Email: elik@gmc.gov.il

Submitted: 24 October 2019
Approved: 05 November 2019
Published: 06 November 2019

How to cite this article: Karra N, Ganam S, Bickel A, Bez M, Abu Shakra I, et al. A unique case of metastatic spinal epidural abscess associated with liver abscess following ascending cholangitis and Escherichia coli bacteremia. Arch Surg Clin Res. 2019; 3: 072-076.

DOI: dx.doi.org/10.29328/journal.ascr.1001039

ORCiD: orcid.org/0000-0002-8796-9724

Copyright: @ 2019 Karra N, et al. This is an open access article distributed under the Creative Commons Attribution License, which permits unrestricted use, distribution, and reproduction in any medium, provided the original work is properly cited.

Check for updates

\section{Introduction}

Spinal epidural abscess (SEA) is a relatively rare infection, with severe potential complications. A retrospective study of a large academic hospital in the US reported an incidence of SEA of 5.1 cases per 10,000 admissions [1]. SEA is an elusive medical condition, with a non-specific clinical presentation. Thus, its diagnosis is often delayed, resulting in neurologic, sometimes irreversible, deficits.

Bacteria may spread to the epidural space by one of three routes: hematogenously, from a contiguous site of infection (psoas or vertebra) or via direct inoculation of the spinal canal during spinal or epidural procedures. The most common mechanism is hematogenous spread from a distant source (skin, the respiratory system or a urinary tract infection), and accounts for $50 \%$ of cases [2]. In approximately two-thirds of cases of bacteremia, the causative agent is Staphylococcus Aureus. Other less common pathogens include gram negative bacilli (16\%), Streptococcus (7\%), coagulase-negative staphylococci and anaerobes [3,4]. In two-thirds of SEA, a port of infection entry may be found. The most commonly identified origins are the skin and soft tissues, and procedures involving the spinal or epidural areas. Many SEAs originate from pyogenic diskitis [5]. However, in about $30 \%$ of SEA, the source of infection remains unidentified. Major risk factors for SEA include intravenous drug use, diabetes mellitus, alcoholism, epidural catheters and trauma [2].

Liver abscess is the most common type of visceral abscesses. The annual incidence is 2.3 cases per 100,000 people according to a retrospective study conducted in the UK [6]. A particularly high rate of pyogenic liver abscesses (PLA) was reported in Southeast Asia [7]. PLA is a life-threatening infection with a mortality rate that may reach $12 \%$ [6]. Commonly described predisposing factors for PLA are diabetes mellitus, an underlying hepatobiliary or pancreatic disease, liver transplant and chronic treatment with protonpump inhibitors [6,8-10]. A possible complication of PLA is metastatic spread, resulting in distant seeding of infection in other organs. 
Here we present a unique case of SEA associated with a hematogenous spread of an Escherichia coli (E. coli) infection originating from a PLA. We also discuss the current literature and other documented cases.

\section{Case Report}

A 71-year-old Jewish male presented to the emergency room with a two-day history of epigastric pain that began after a meal, accompanied by nausea and vomiting. There was no recent history of high fever, urinary complaints or diarrhea. His medical history included type 2 diabetes mellitus, hypertension, benign prostatic hypertrophy, chronic back pain and a family history of prostate cancer. At presentation his vital signs were: blood pressure 101/64, pulse 90 and body temperature $36.5{ }^{\circ} \mathrm{C}$. His physical examination was unremarkable except for mild epigastric tenderness, without guarding or rebound. Murphy's sign was negative. His ECG was normal, with no signs of ischemia. Lab tests exhibited leukocytosis with left shift (13.9k white blood cells, $13 \mathrm{k}$ neutrophils), increased hepatocellular enzymes (AST $=316$ $\mathrm{U} / \mathrm{L}, \mathrm{ALT}=250 \mathrm{U} / \mathrm{L})$ and markedly increased lipase (8182 $\mathrm{U} / \mathrm{L}$ ) and amylase (1850 U/L) (Table 1). His lipid profile was within the normal range. An abdominal ultrasonography exhibited a normal size fatty liver and a normal size gallbladder with mildly thickened hyperemic walls, without gallstones. There was no dilatation of the intra- or extrahepatic biliary ducts. The patient was admitted to the surgical department with a diagnosis of non-biliary pancreatitis and conservative treatment was initiated with analgesics and fluids. Upon admission, the patient developed a fever of 38.7 ${ }^{\circ} \mathrm{C}$. His lab tests now revealed worsening leukocytosis and an increased level of C-reactive protein (155 mg/l) (Table 1).

On the second day the patient developed combined

Table 1: Laboratory tests in the emergency room (ER) and over the course of 23 days of hospitalization.

\begin{tabular}{|c|c|c|c|c|c|c|c|c|c|}
\hline & ER & Day 1 & Day 2 & Day 3 & Day 4 & Day 5 & Day 6 & Day 9 & Day 23 \\
\hline $\mathrm{Hb}(\mathrm{g} / \mathrm{dl})$ & 15.8 & 14.2 & 13.2 & 13.2 & 12.3 & 12.5 & 12.1 & 10.8 & 11.4 \\
\hline HCT (\%) & 47.9 & 41.9 & 40.4 & 39.1 & 36.6 & 36.7 & 35.1 & 31.9 & 34.6 \\
\hline PLT $\left(x 10^{3}\right) / \mathrm{ul}$ & 73 & 159 & 162 & 85 & 61 & 52 & 58 & 183 & 378 \\
\hline WBC $\left(x 10^{3}\right) / u l$ & 13.9 & 28.9 & 21.7 & 11.9 & 13.6 & 10.7 & 10.8 & 19.09 & 10.8 \\
\hline $\begin{array}{l}\text { Neu abs. } \\
\left(x 10^{3}\right) / \mathrm{ul}\end{array}$ & 13.06 & 27.23 & 20.6 & 10.3 & 11.9 & 9.4 & 8.8 & 19.14 & 7.3 \\
\hline $\mathrm{CRP}(\mathrm{mg} / \mathrm{l})$ & 4 & 155 & 173 & 252 & 297 & 238 & 221 & 121 & 23.2 \\
\hline AST (U/L) & 316 & NA & 389.10 & 66.6 & 63 & 43 & 45.7 & 25.3 & 23.1 \\
\hline $\mathrm{ALT}(\mathrm{U} / \mathrm{L})$ & 250 & 735 & 654.6 & 254.5 & 187.40 & 142 & 97.8 & 40.8 & 23.5 \\
\hline ALP (U/L) & 111 & 133 & 137 & 196 & 170 & 166 & 173 & 132 & 79 \\
\hline GGT (U/L) & NA & NA & 566 & 361.9 & 275.9 & 231.7 & 242 & 201.8 & NA \\
\hline $\begin{array}{l}\text { Bil. Total (mg/ } \\
\text { dl) }\end{array}$ & 1.51 & 1.36 & 1.28 & 2 & 2.6 & 2.04 & 1.37 & 0.77 & 0.32 \\
\hline $\begin{array}{l}\text { Bil. Direct (mg/ } \\
\text { dl) }\end{array}$ & 1.11 & 0.75 & 0.89 & 1.56 & 2.01 & 1.54 & 1.04 & NA & NA \\
\hline Amylase (U/L) & 1850 & NA & NA & 88.6 & NA & NA & 76.7 & NA & NA \\
\hline Lipase (U/L) & 8182 & 631.2 & 163 & 155.3 & 143.5 & NA & 108.3 & 205.7 & NA \\
\hline $\begin{array}{l}\text { Calcium (mg/ } \\
\text { dl) }\end{array}$ & 10.5 & NA & 8.2 & 8.38 & 8.17 & NA & 8.02 & NA & NA \\
\hline Albumin (g/dl) & 4.73 & 4.73 & 3.81 & 3.37 & 3.16 & 3.05 & 2.92 & 2.8 & NA \\
\hline
\end{tabular}

NA: Not Available.

hepatocellular and cholestatic dysfunction with hyperbilirubinemia, while his lipase decreased to $163 \mathrm{U} / \mathrm{L}$. Physical examination showed right upper quadrant tenderness. A diagnosis of cholangitis was presumed. Chest X-ray and urine cultures were normal. Blood cultures were positive for non-ESBL E. coli. Thus, ceftriaxone and metronidazole treatments were initiated. The next day (day 3) the patient was still febrile, with chills and abdominal pain, and his C-reactive protein level had escalated to $300 \mathrm{mg} / \mathrm{l}$. Since gallstones were not initially found on ultrasound, we decided to perform an abdominal CT and to proceed with endoscopic ultrasound and endoscopic retrograde cholangiopancreatography if appropriate. CT revealed minimal bilateral pleural effusions but without any other pathological findings: the size, shape and density of the gallbladder, liver, biliary tracts, spleen and pancreas were normal (Figure 1A). Similarly, the endoscopic ultrasound demonstrated a slightly thickened gallbladder and a $6 \mathrm{~mm}$ common bile duct, without evidence of cholelithiasis or choledocholithiasis.

During the next couple of days, laboratory values began to normalize; however, complaints of abdominal pain persisted and the patient was still febrile, with peaks of fever at nights. Therefore, CT was repeated on day 9 , revealing a $5.5 / 4.3 \mathrm{~cm}$ hypodense irregular lesion at segment 4 of the liver, with a thrombus in the adjacent portal branch (Figure 1B,C). To further characterize the findings, an abdominal MRI was completed on day 12, which showed an extensive irregular process, enhanced at $\mathrm{T} 2$, and comprising multiple small cystic spaces at segments 2, 4 and 8 of the liver (Figure 1D). These finding were consistent with micro PLA. A thrombus was also imaged in a left portal branch, as already shown on CT. A biopsy was taken from the lesions. However, the cultures returned negative. We initiated anticoagulation with low molecular weight heparin, and the antibiotic treatment was continued.

On the ninth day of admission, the patient began to complain about low back pain, paresthesia in the lower
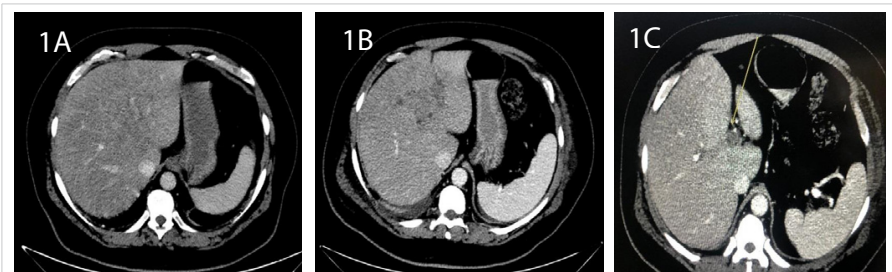

$1 \mathrm{D}$
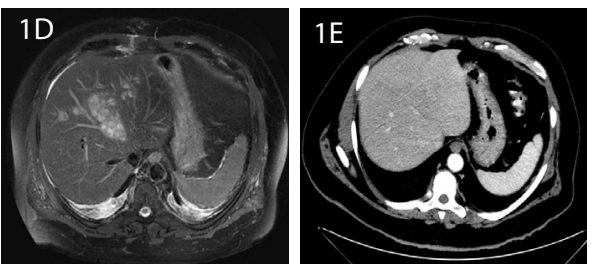

Figure 1: CT images of the liver: 1A: Day 3. The shape and size are normal, with no pathological findings. 1B: Day 9. A hypodense irregular lesion is observed. $1 \mathrm{C}$ Thrombus of the portal branch 1D: Abdominal MRI shows an extensive irregular process enhanced at T2, with multiple small cystic spaces at segments 2,4 and 8 . $1 \mathrm{E}$ : At day 24 , the micro-abscesses are regressed following antibiotic treatment. 
limbs and difficulty walking due to pain. There were no motor or sensory deficits on his neurological exam. Initially, musculoskeletal pain was presumed since he was known to have chronic back pain. However, complaints persisted during the next days and he developed focal tenderness in the lumbar area. Thus, a spinal cord MRI was performed on day 16, which exhibited a non-homogenous 5.3/1 cm tissue in the spinal canal at level L5-S1, posterior to the vertebras and surrounding the dural sac (Figure 2). Following contrast injection, an enhanced signal was evident at level L5-S1, at the left wing of the sacrum, paravertebral tissue and intervertebral disc. A diagnosis of SEA with diskitis and osteomyelitis was established. The patient was transferred to the orthopedic department and was urgently operated. Laminotomy, phlebectomy, separation of loculations and irrigations were performed. Cultures from the drained pus returned negative. During the subsequent days, drained secretions decreased to a minimum, the patient's condition gradually improved and his back pain and paresthesia resolved. Furthermore, his lab tests normalized (Table 1). A follow up CT performed at day 24 exhibited almost complete regression of the liver abscesses (Figure 1E).

\section{Discussion}

We present a patient with PLA, with two known predisposing risk factors for this infection, namely, diabetes and cholangitis. PLAs are mostly polymicrobial, with mixed enteric facultative and anaerobic pathogens. Enteric gramnegative bacilli, specifically $E$. coli and Klebsiella pneumonia (K. pneumoniae) are the most commonly identified bacteria [11]. E. coli bacteremia was identified in the described patient. Until the 1980s, E. coli was the most commonly isolated pathogen from PLAs in the US [12]. However, a shift in recent years shows that K. pneumonia has become highly endemic in South Asia, as well as in North America [13]. High rates of subsequent bacteremia have been reported in PLA, resulting in metastatic spread to other distant organs, particularly when $K$. pneumoniae was the implicated pathogen [14].

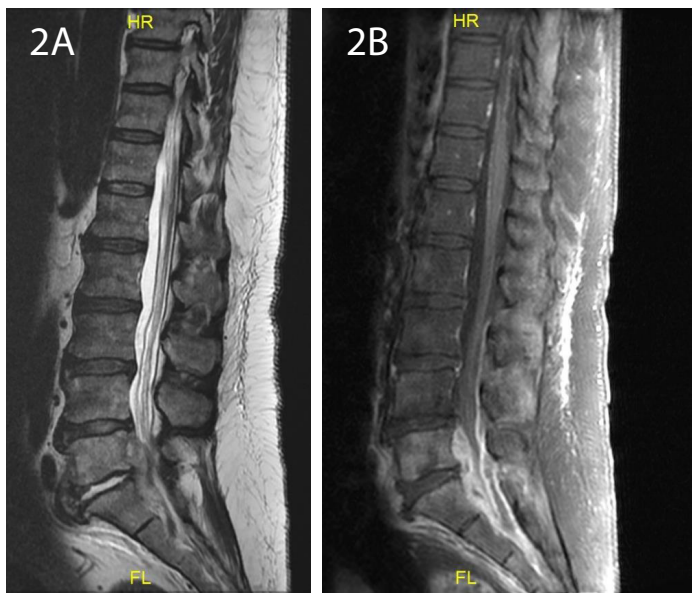

Figure 2: A. Spinal cord MRI: A non-homogenous tissue in the spinal canal at leve L5-S1, posterior to the vertebras. B: Following contrast injection, an enhanced signal was evident at the L5-S1 level.
Indeed, a retrospective Taiwanese study with a 1-year follow up period showed a higher hazard ratio among persons with than without PLA, for developing metastatic infections: pulmonary abscess, empyema, renal and perinephric abscess, splenic abscess and SEA [15]. Notably, in 73\% of PLAs the causative pathogen was $K$. pneumoniae, and the results were further corroborated when the analysis was restricted to $K$. pneumoniae PLAs [15]. A distinct syndrome caused by strains of $K$. pneumoniae bearing the hypermucoviscous phenotype mediated by the $\mathrm{K} 1$ or $\mathrm{K} 2$ capsule was reported to cause high rates of endophtalmitis and CNS infections [16]. Similarly, even though epidural abscess is considered a rare complication, at least three case reports have described the association of a metastatic $K$. pneumoniae bacteremia and liver abscess to the occurrence of a cervical abscess or SEA $[17,18]$.

Though extremely rare, cases of SEA caused by E coli following urinary tract infections have been reported $[19,20]$. To the best of our knowledge, this is the first case report describing SEA due to E. coli bacteremia following a PLA.

Notably, though E. coli was isolated from blood cultures drawn at day 2, cultures taken from the liver abscesses and epidural collection on days 9 and 16, respectively, were negative. This is most likely attributed to the antibiotic treatment regimen that was initiated more than one week prior to the sampling of liver and epidural abscesses. The liver micro-abscesses were successfully treated by appropriate intravenous antibiotics, without the need for surgical or percutaneous drainage. This is due to the fact that lesions were multiple and too small for intervention.

Contrast CT and ultrasound are typically used to diagnose liver abscess, with higher sensitivity in the former [21]. However, CT may not always detect micro-abscesses or distinguish them from diffuse fatty infiltration of the liver parenchyma or from liver metastasis [22]. Indeed, in the first CT performed on day 3 in our patient, micro-abscesses were not visualized. Only on day 7 were the lesions observed. In such cases, contrast MRI is considered more accurate in defining and characterizing the findings [23]. Truly, a subsequent MRI established the diagnosis of micro-abscesses in the present case.

Another interesting finding in our patient was thrombosis of a portal vein branch, observed both on CT and on MRI. This complication has also been documented in the literature, particularly in association with $K$. pneumoniae liver abscess, with a remarkable $30 \%$ rate of cases $[24,25]$. Interestingly, septic thrombophlebitis has also been associated with higher incidence of hematogenous septic complications, suggesting that thrombophlebitis may be involved in the dissemination of the infectious processes [24]. This said, some studies suggest a spontaneous resolution of both hepatic and portal vein septic thromboses associated with $K$. pneumoniae PLA, 
by targeting treatment to the underlying abscess without the need for anticoagulation [26,27]. Nevertheless, data are insufficient to support this approach as a definite consensus. Further, anticoagulation treatment in the present case was initiated prior to establishing a diagnosis of micro-abscesses by MRI.

While, back pain, fever and neurologic deficits are the most common presenting symptoms of SEA, they rarely occur together. Often, symptoms gradually progress, as described by Heusner [28]: from focal severe back pain, root pain in the distribution of the affected nerve root, motor weakness with bladder or bowel dysfunction, and finally to paralysis. The mortality rate of SEA is $5 \%$, mostly due to overwhelming sepsis [2]. Neurologic recovery strongly correlates with the duration of the neurologic symptoms and becomes very unlikely if paralysis presents for more than 2448 hours prior to surgery [2]. Unfortunately, the prognosis of epidural abscess is often hurdled due to a significant delay in diagnosis.

Though our patient complained of back pain and paresthesia, his physical examination did not show any motor or other neurologic deficits. It is noteworthy that a very early diagnosis of SEA was established, with prompt and efficacious treatment. Both a high index of clinical suspicion and an early performance of MRI account for this successful outcome.

The management of SEA consists of surgical intervention combined with 4-8 weeks of antibiotic treatment. If osteomyelitis also presents, a 6-8-week regimen is required. Follow-up MRI should be performed at 4-6 weeks. At the writing of this paper, our patient has been treated with 4 weeks of ceftriaxone with the intention to complete 6 weeks of antibiotic therapy. With this treatment he has dramatically improved and is on the way to complete recovery with no neurologic sequelae.

\section{Conclusion}

We describe a unique case of $E$. coli liver micro-abscesses and portal vein thrombosis with subsequent bacteremia and spinal epidural abscess. Although similar cases have been described with K. pneumoniae, this is the first reported case of SEA secondary to E. coli PLA. SEA was successfully treated with surgical intervention and antibiotic treatment. While SEA is a rare medical entity, the potential outcomes could be catastrophic without a timely treatment. Thus, this case underscores the importance of physician awareness to SEA, to its early diagnosis and emergency intervention.

\section{References}

1. Vakili M, Crum-Cianflone NF. Spinal Epidural Abscess: A Series of 101 Cases. Am J Med. 2017; 130: 1458-1463.

PubMed: https://www.ncbi.nlm.nih.gov/pubmed/28797646

2. Darouiche RO. Spinal Epidural Abscess. N Engl J Med. 2006; 355 : 2012-2020.

PubMed: https://www.ncbi.nlm.nih.gov/pubmed/17093252
3. Darouiche RO, Hamill RJ, Greenberg SB, Weathers SW, Musher DM. Bacterial spinal epidural abscess. Review of 43 cases and literature survey. Medicine (Baltimore). 1992; 71: 369-385. PubMed: https://www.ncbi.nlm.nih.gov/pubmed/1359381

4. Reihsaus E, Waldbaur H, Seeling W. Spinal epidural abscess: a metaanalysis of 915 patients. Neurosurg Rev. 2000; 23: 175-205. PubMed: https://www.ncbi.nlm.nih.gov/pubmed/11153548

5. Kapeller P, Fazekas F, Krametter D, Koch M, Roob G, et al. Pyogenic infectious spondylitis: clinical, laboratory and MRI features. Eur Neurol. 1997; 38: 94-98.

PubMed: https://www.ncbi.nlm.nih.gov/pubmed/9286631

6. Mohsen AH, Green ST, Read RC, McKendrick MW. Liver abscess in adults: ten years' experience in a UK centre. QJM. 2002; 95: 797-802. PubMed: https://www.ncbi.nlm.nih.gov/pubmed/12454322

7. Shi SH, Feng XN, Lai MC, Kong HS, Zheng SS. Biliary diseases as main causes of pyogenic liver abscess caused by extended-spectrum betalactamase-producing Enterobacteriaceae. Liver International. 2017; 37: 727-734.

PubMed: https://www.ncbi.nlm.nih.gov/pubmed/27718321

8. Thomsen RW, Jepsen P, Sørensen HT. Diabetes mellitus and pyogenic liver abscess: risk and prognosis. Clin Infect Dis. 2007; 44: 1194-1201. PubMed: https://www.ncbi.nlm.nih.gov/pubmed/17407038

9. Chan KS, Chen CM, Cheng KC, Hou CC, Lin HJ, et al. Pyogenic liver abscess: a retrospective analysis of 107 patients during a 3-year period. Jpn J Infect Dis. 2005; 58: 366-368.

PubMed: https://www.ncbi.nlm.nih.gov/pubmed/16377869

10. Lin HF, Liao KF, Chang CM, Lin CL, Lai SW. Correlation between proton pump inhibitors and risk of pyogenic liver abscess. Eur J Clin Pharmacol. 2017; 73: 1019-1025.

PubMed: https://www.ncbi.nlm.nih.gov/pubmed/28434021

11. Johannsen EC, Sifri CD, Madoff LC. Pyogenic liver abscesses. Infect Dis Clin North Am. 2000; 14: 547-563.

PubMed: https://www.ncbi.nlm.nih.gov/pubmed/10987109

12. Lazarchick J, DeSouza NA, Nichols DR, Washington II JA. Pyogenic liver abscess. Mayo Clin Proc 1973; 48: 349-355.

PubMed: https://www.ncbi.nlm.nih.gov/pubmed/4703302

13. Pope JV, Teich DL, Clardy P, McGillicuddy DC. Klebsiella pneumoniae liver abscess: an emerging problem in North America. J Emerg Med. 2011; 41: e103-e105.

PubMed: https://www.ncbi.nlm.nih.gov/pubmed/18993020

14. Leederman E, Crum N. Pyogenic liver abscess with a focus on klebsiella pneumoniae as a primary pathogen: an emerging disease with unique clinical characteristics. Am J Gastroenterol. 2005; 100: 322-331. PubMed: https://www.ncbi.nlm.nih.gov/pubmed/15667489

15. Keller JJ, Tsai MC, Lin CC, Lin YC, Lin HC. Risk of infections subsequent to pyogenic liver abscess: a nationwide population-based study. Clin Microbiol Infect. 2013; 19: 717-722.

PubMed: https://www.ncbi.nlm.nih.gov/pubmed/23034092

16. Fang CT, Lai SY, Yi WC, Hsueh PR, Liu KL, et al. Klebsiella pneumoniae genotype K1: an emerging pathogen that causes septic ocular or central nervous system complications from pyogenic liver abscess. Clin Infect Dis. 2007; 45: 284-293.

PubMed: https://www.ncbi.nlm.nih.gov/pubmed/17599305

17. Kuramochi G, Takei S, Sato M, Isokawa $O$, Takemae T, et al. Klebsiella pneumoniae liver abscess associated with septic spinal epidural abscess. Hepatol Res. 2005; 31: 48-52.

PubMed: https://www.ncbi.nlm.nih.gov/pubmed/15652471

18. Hsieh MJ, Lu TC, Ma MH M, Wang HP, Chen SC. Unrecognized cervical spinal epidural abscess associated with metastatic klebsiella 
pneumoniae bacteremia and liver abscess in non-diabetic patients. Diagn Microbiol Infect Dis 2009; 65: 65-68.

PubMed: https://www.ncbi.nlm.nih.gov/pubmed/19679238

19. O'Neill SC, Baker JF, Ellanti P, Synnott K. Cervical epidural abscess followinganEscherichiacoliurinarytractinfection. BMJCaseRep.2014. PubMed: https://www.ncbi.nlm.nih.gov/pubmed/24473426

20. Moustafa A, Kheireldine R, Khan Z, Alim H, Khan MS, et al. Cervical Spinal Osteomyelitis with Epidural Abscess following an Escherichia coli Urinary Tract Infection in an Immunocompetent Host. Case Rep Infect Dis. 2019; 2019: 5286726.

PubMed: https://www.ncbi.nlm.nih.gov/pubmed/31143482

21. Halvorsen RA, Korobkin M, Foster WL, Silverman PM, Thompson WM. The variable CT appearance of hepatic abscesses. Am J Roentgenol. 1984; 142: 941-946.

PubMed: https://www.ncbi.nlm.nih.gov/pubmed/6372412

22. Wang N, Ju Y, Wu J, Liu A, Chen A, et al. Differentiation of liver abscess from liver metastasis using dual-energy spectral CT quantitative parameters. Eur J Radiol. 2019; 113: 204-208.

PubMed: https://www.ncbi.nlm.nih.gov/pubmed/30927948

23. Choi SY, Kim KY, Min JH, Cha DI, Jeong WK, et al. The value of gadoxetic acid-enhanced MRI for di?erentiation between hepatic microabscesses and metastases in patients with periampullary cancer, Eur. Radiol. 2017; 27: 4383-4393.

PubMed: https://www.ncbi.nlm.nih.gov/pubmed/28342102

24. Alsaif HS, Venkatesh SK, Chan DS, Archuleta S. CT appearance of pyogenic liver abscesses caused by Klebsiella pneumoniae. Radiology. 2011; 260: 129-138.

PubMed: https://www.ncbi.nlm.nih.gov/pubmed/21460028

25. Syed MA, Kim TK, Jang HJ. Portal and hepatic vein thrombosis in liver abscess: CT findings. Eur J Radiol. 2007; 61: 513-519. PubMed: https://www.ncbi.nlm.nih.gov/pubmed/17161932

26. Wang YF, Chang CC, Lee TC, Shih IL, Lien WC, et al. Recent trend of pylephlebitis in Taiwan: Klebsiella pneumoniae liver abscess as an emerging etiology. Infection. 2013; 41: 1137-1143.

PubMed: https://www.ncbi.nlm.nih.gov/pubmed/23817997

27. Molton JS, Chee YL, Hennedige TP, Venkatesh SK, Archuleta S. Impact of Regional Vein Thrombosis in Patients with Klebsiella pneumoniae Liver Abscess. PLoS One. 2015; 10: e0140129. PubMed: https://www.ncbi.nlm.nih.gov/pubmed/26444702

28. Heusner AP. Nontuberculous spinal epidural infections. N Engl J Med. 1948; 239: 845-854.

PubMed: https://www.ncbi.nlm.nih.gov/pubmed/18894306 